\title{
Does kidney transplantation onto the external iliac artery affect the haemodynamic parameters of the cavernosal arteries?
}

\author{
Paolo Gontero ${ }^{1}$, Marco Oderda ${ }^{1}$, Claudia Filippini ${ }^{2}$, Francesco Fontana ${ }^{3}$, Elisa Lazzarich ${ }^{4}$ Piero Stratta ${ }^{4}$, \\ Ernesto Turello ${ }^{4}$, Alessandro Tizzani ${ }^{1}$ and Bruno Frea ${ }^{5}$
}

Reduced cavernosal arterial inflow has been hypothesized to be the likely cause of erectile dysfunction after kidney transplants in recipients revascularized through end-to-end anastomosis to the internal iliac artery, suggesting that end-to-side anastomosis at the external iliac artery is preferable. The aim of this study was to prospectively evaluate the effect of the use of the external iliac artery on erectile function, hormone profiles and penile blood flow by evaluating changes in penile colour Doppler ultrasound parameters in a consecutive series of 22 recipients before and after end-to-side external iliac artery transplantation. The mean International Index of Erectile Function-Erectile Function (IIEF-EF) domain score decreased significantly 3 months after transplant $(18.09 \pm 6.33 \mathrm{vs}$. $22.50 \pm 7.09, P=0.01$ ). The reduction in peak systolic velocity (PSV) was significant for the cavernous artery homolateral to the side of transplant $(42.60 \pm 18.77$ vs. $52.01 \pm 19.91, P=0.01)$. The mean postoperative end diastolic velocity (EDV) did not differ significantly from the preoperative value $(P=0.74)$. No statistical differences were found in the serum levels of testosterone or prolactin. Kidney grafts anastomosed at the external iliac artery produced significant $(P=0.01)$ reductions in arterial inflow at the homolateral cavernosal artery that remained above the normal threshold. Whether these haemodynamic changes can explain the worsening of postoperative erectile function remains to be proven.

Asian Journal of Andrology (2012) 14, 621-625; doi:10.1038/aja.2011.115; published online 26 December 2011

Keywords: cavernosal arteries; Doppler; erectile dysfunction; external iliac artery; kidney transplant

\section{INTRODUCTION}

End-stage renal disease (ESRD) is significantly related to erectile dysfunction (ED). ${ }^{1}$ The incidence of $\mathrm{ED}$ in men with chronic renal failure is estimated to be approximately $70 \%,{ }^{2}$ with a prevalence of up to $82 \%$ in haemodialysis patients. ${ }^{3}$ The aetiology of ED in ESRD patients is often multifactorial. ${ }^{4}$ At present, kidney transplantation (KT) has become a widely used therapy for ESRD, and the number of transplants performed is increasing, allowing the restoration of normal kidney function. But what happens to erectile function? While several studies have suggested that erectile function significantly improves after successful renal transplantation, ${ }^{5-8}$ especially when the surgery is performed at a younger age, ${ }^{5}$ other studies have shown minimal effects of transplantation on erectile performance or even deterioration. $^{9-12}$ The overall prevalence of ED after KT remains high, ranging from $48.9 \%{ }^{13}$ to $55.7 \% .{ }^{11}$ The potential causative effects of $\mathrm{ED}$ in transplant recipients may include anxiety, medication side effects, impaired penile vascularity and failure to resolve hormonal abnormalities or underlying disease comorbidities. ${ }^{14}$ Reduced cavernosal arterial inflow was hypothesized as the likely cause of ED in a series in which the internal iliac artery was employed for end-to-end anastomosis. ${ }^{14,15}$
One study observed a significant decrease in the dynamic peak systolic velocity (PSV) and resistance index (RI) in impotent recipients revascularized through end-to-end anastomosis to the internal iliac artery compared to end-to-side anastomosis to the external iliac artery, suggesting that unilateral ligation of the internal iliac artery may play a negative role in erectile function following KT. ${ }^{16}$ Hence, end-to-side anastomosis of the graft to the external iliac artery has been recommended, particularly in the presence of associated vascular risk factors that could compromise pelvic blood flow. ${ }^{14}$ However, the prevalence of ED remains high, even in modern series of KT recipients onto the external iliac artery. ${ }^{12}$ It is difficult to evaluate the real effect of the artery used for anastomosis on penile blood flow, especially considering that sometimes the blood supply is also provided by accessory arteries that arise from the external iliac, either directly or through its obturator or vesical branches. ${ }^{17}$ To our knowledge, at the present time, there are no studies that specifically compare cavernosal haemodynamic parameters before and after KT onto the external iliac artery. The primary aim of the present study was to prospectively evaluate changes in penile colour Doppler ultrasound (CDUS) parameters in a consecutive series of renal transplant recipients before and after 
end-to-side external iliac artery transplantation in order to evaluate the effect of the use of the external iliac artery on erectile function and penile blood flow. The postoperative changes in hormone profiles were taken as a secondary end point.

\section{MATERIALS AND METHODS}

\section{Patients and inclusion criteria}

This was a prospective study conducted on consecutive male patients suffering from ESRD who were on haemodialysis; the patients were enrolled the day they were admitted in the hospital for a kidney transplant. The inclusion criteria included the absence of any acute or concomitant disease or infection contraindicating a kidney transplant, a history of $\geqslant 1$ year of haemodialysis and being sexually active or interested in regaining sexual activity. Previous or concurrent treatment for ED, penile deformities or nodules and any potential neurogenic cause of ED (including previous pelvic surgery) constituted the exclusion criteria. The eligible patients were required not to start any ED treatment until the 3-month postoperative evaluation. Institutional board approval was not required for the development of this study at our centre (Maggiore della Carità Hospital, Novara, Italy).

\section{Baseline (preoperative) investigations}

From 2000 to 2003, 35 patients were screened for the study on the day their kidney transplants were scheduled. Eight declined to take part in the study. Three were excluded because they did not fulfil the inclusion criteria (two due to a lack of interest in regaining sexual activity and one because of concomitant multiple sclerosis). The 24 remaining patients agreed to enter the study and signed an informed consent agreement. The aetiology of ESRD and any related or unrelated comorbidities that emerged through a complete medical history were recorded in a database. A serum sample was stored for the determination of testosterone and prolactin levels. Erectile function was assessed on the basis of the score on the six questions from the erectile function domain of the International Index of Erectile Function-Erectile Function (IIEF-EF) and ED and categorized according to Cappelleri et al. ${ }^{18}$ into: absent ED (EF score, 26-30), mild ED (EF score, 17-25), moderate ED (EF score, 11-16) and severe ED (EF score, $\leqslant 10$ ).

All patients underwent a penile dynamic CDUS performed using an Acuson 128 X P/10 (Acuson, Mountain View, CA, USA) with a 7 - to $10-\mathrm{MHz}$ extended frequency linear array transducer, performed by an operator blinded to the purpose of the study. Erections were induced by an intracavernous injection of $20 \mu \mathrm{g}$ prostaglandin E1, and serial measurements were taken every 5 min for 20 min of the PSV, end diastolic velocity (EDV) and RI. The RI was calculated as (PSV-EDV)/PSV. Generally accepted normal values were used: dynamic PSV $<30 \mathrm{~cm} \mathrm{~s}^{-1}$ was indicative of arterial insufficiency and $\mathrm{RI}<0.85$ and $\mathrm{EDV}>5 \mathrm{~cm} \mathrm{~s}^{-1}$ was suggestive of veno-occlusive dysfunction. ${ }^{16}$ The PSVs of both cavernosal arteries were recorded separately. Prostaglandin E1-induced erection was categorized into four grades according to objective examination
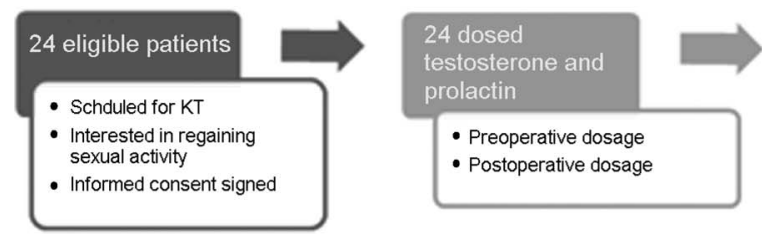

criteria: ${ }^{19}$ grade I was defined as partial tumescence with inadequate rigidity for penetration; grade II was defined as full tumescence with moderate rigidity; grade III was defined as complete tumescence with 'suboptimal' rigidity (sufficient for penetration but inadequate for satisfactory sexual intercourse); and grade IV was defined as full rigidity lasting at least $20 \mathrm{~min}^{20}$

Surgery was performed according to the standard institutional technique, with the graft anastomosed end to side to the external iliac artery in all cases. The recipient side was right in 10 patients and left in 14. All patients received an immunosuppressive regimen according to the standard protocol in use.

\section{Postoperative investigations}

CDUS was repeated on the day of discharge, generally the twentieth postoperative day, after catheter removal. At 3 months postoperatively, a serum sample for testosterone and prolactin was sent and the EF domain of the IIEF was administered.

\section{End points and statistics}

The primary study endpoint was the assessment of changes in the penile CDUS parameters of renal transplant recipients with anastomosis of the graft onto the external iliac artery before and after the surgery. The CDUS parameters were also compared between patients with postoperative worsening of the EF domain of the IIEF and patients with no worsening and between the cavernosal artery homolateral to the side of the kidney transplant and the artery contralateral to the side of the transplant. Changes in the serum concentrations of prolactin and testosterone before and after the transplant were taken as secondary end points.

Statistical analysis was performed using a two-tailed paired sample $t$-test, using SAS software, release 8.02 (SAS Institute Inc., Cary, NC, USA). $P<0.05$ was considered statistically significant. In cases of comparisons of proportions, a Chi-square test was used to test the statistical significance.

\section{RESULTS}

Among the 24 consecutive patients, aged 23-68 years (mean \pm s.d.: $50.62 \pm 12.05$ ), one refused to undergo the preoperative CDUS and was excluded from study. Of the remaining 23 patients, one failed to complete the 3-month postoperative IIEF-EF domain questionnaire. Figure 1 shows the flow chart of the study. The patients' comorbidities, the aetiology of ESRD and the immunosuppressive regimen adopted postoperatively are shown in Table 1 . The mean duration of ESRD (time of dialysis) was $6.25 \pm 2.96$ (range: $2-11$ years).

Table 2 displays the EF domain scores of the IIEF as mean values and categorized into degrees of ED. The mean maximal in-office erection grade values after prostaglandin administration at baseline and at catheter removal are also reported in Table 2. Overall, the rate of subjects with $\mathrm{EF}$ domain scores consistent with $\mathrm{ED}$ increased from $45 \%$ preoperatively to $91 \%$ at 3 months postoperatively $(P=0.003)$, with a significant proportion of patients developing mild ED $(59.1 \%$
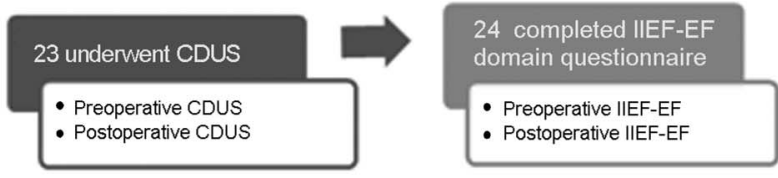

Figure 1 Flow chart of the study. 
Table 1 Baseline patient characteristics $(N=24)$

\begin{tabular}{lc}
\hline Parameter & Value (\%) \\
\hline Age, year (mean \pm s.d.) & $50.62 \pm 12.05$ \\
Vascular risk factors, $\boldsymbol{n}$ (\%) & \\
Smoking & $13(54.2)$ \\
Hypertension & $19(79.2)$ \\
Coronary artery disease & $2(8.3)$ \\
Diabetes & $1(4.2)$ \\
Aetiologies of chronic renal failure in the cohort, $\boldsymbol{n}(\%)$ & \\
Chronic glomerulonephritis & $2(8.3)$ \\
Glomerular sclerosis & $2(8.3)$ \\
Polycystic kidney disease & $6(25.0)$ \\
IgA nephropathy (Berger's disease) & $5(20.8)$ \\
Hereditary nephropathy & $2(8.3)$ \\
Unknown & $7(29.2)$ \\
Immunosuppressive regimen, $\boldsymbol{n}$ (\%) & \\
Tacrolimus-Prednisolone & $2(8.3)$ \\
Tacrolimus-Prednisolone-Mycophenolate mofetil & $17(70.8)$ \\
Tacrolimus-Prednisolone-Sirolimus & $2(8.3)$ \\
Cyclosporine A-Prednisolone-Mycophenolate mofetil & $1(4.2)$ \\
Sirolimus-Prednisolone-Mycophenolate mofetil & $1(4.2)$ \\
Prednisolone-Mycophenolate mofetil & $1(4.2)$ \\
\hline
\end{tabular}

vs. $22.7 \%, P=0.03)$. The mean IIEF-EF domain score for the entire cohort significantly decreased 3 months after the transplants compared to baseline $(22.50 \pm 7.09$ vs. $18.09 \pm 6.33, P=0.01)$. The decrease in mean dynamic postoperative erection grading was of borderline statistical significance $(3.11 \pm 0.91$ vs. $2.79 \pm 0.84, P=0.06)$. Testosterone levels below the $200 \mathrm{ng} \mathrm{ml}^{-1}$ normality threshold were recorded in three subjects preoperatively and in one subject postoperatively. No patient showed elevated prolactin levels, either preoperatively or postoperatively. No statistical differences were found between the baseline and 3-month postoperative serum levels of testosterone (409.95 \pm 181.87 vs. $419.04 \pm 129.09, P=0.68)$ and prolactin (16.22 \pm 35.04 vs. $10.54 \pm 9.00, P=0.38$ ).

At CDUS evaluation (Table 3), abnormal dynamic PSV values were reported preoperatively in six $(26 \%)$ and postoperatively in eight (35\%) out of 23 patients ( $P=0.73$ ). The mean PSV of the cavernous artery homolateral to the side of transplant was significantly reduced postoperatively compared with the preoperative mean $(42.60 \pm 18.77$ vs. $52.01 \pm 19.91, P=0.01)$. The mean PSV of the right cavernous artery was also significantly reduced $(42.94 \pm 13.63$ vs. $50.72 \pm 16.45$, $P=0.04$ ), while the mean PSV of the left artery was reduced, but not significantly ( $45.13 \pm 17.95 v s .48 .07 \pm 21.16, P=0.43)$. The mean postoperative EDV did not differ significantly from the preoperative value (5.62 \pm 3.17 vs. $5.98 \pm 3.07, P=0.74)$, and neither did the mean RI $(0.88 \pm 0.10$ vs. $0.88 \pm 0.08, P=0.94)$. Significant reductions in the mean PSV at the transplant site were documented in patients reporting a postoperative reduction of at least four points in the EF domain of the IIEF but not in those patients with a reduction of less than four points in the $\mathrm{EF}$ domain score ( $P=0.02$ and $P=0.09$, respectively).

We performed a multivariate analysis taking into account age, smoking, diabetes, $\mathrm{CAD}$ and hypertension in order to evaluate their impact on sexual function. None of these factors, however, were significantly associated with the outcome ( $P>0.05$, data not shown), probably because our study was underpowered to detect such an association.

\section{DISCUSSION}

The main results of our study showed that KT onto the external iliac artery causes early worsening of erectile function, based on IIEF-EF scores, and significant reductions in penile arterial inflow, although above the normality threshold. It would seem that the type of artery chosen for KT plays hardly any role in cavernosal artery haemodynamics. ESRD patients on haemodialysis have a high prevalence of ED, an additional detriment to their already impaired quality of life. The beneficial effect of $\mathrm{KT}$ in restoring sexual function, advocated by some studies, ${ }^{5-8}$ remains a highly controversial issue. According to some authors, patients show significant improvement in ED, based on IIEF scores, after KT, ${ }^{5,8}$ especially when they are younger and have less severe ED. Conversely, other reports have suggested that as many as $48.9 \%{ }^{13}$ to $55.7 \%{ }^{11}$ of recipients continue to suffer ED. ElBahnasawy et al. ${ }^{9}$ found that erectile function improved in $40 \%$ of patients but deteriorated or remained unchanged in $18 \%$ and $42 \%$, respectively, of patients 6 months post-transplantation. Notably, in a recent study of $78 \mathrm{KT}$ recipients assessed with the IIEF, the ED rate increased from $87 \%$ preoperatively to $91 \%$ one year postoperatively, with a mean reduction of the EF domain from 18.48 to $17.55 .{ }^{12}$ In line with these latter findings, the EF domain of the IIEF significantly decreased $(P=0.01) 3$ months after KT compared to the baseline value, and the ED rate rose from $45 \%$ preoperatively to $91 \%$ postoperatively in our prospective cohort. Several causal factors have been suggested, such as anxiety, medication side effects, impaired penile vascularity and failure to resolve hormonal abnormalities or underlying disease processes. ${ }^{14}$ Haemodynamic changes in the cavernosal arteries following KT have been hypothesized as a likely cause of ED. This effect may be particularly true when the renal graft is anastomosed end to end to the internal iliac artery, thus diverting the blood inflow from the internal pudendal artery. The risk of vasculogenic impotence following end-to-end anastomosis of the renal graft was reported to be $10 \%$ in an early study. ${ }^{15}$ In a retrospective CDUS study, Abdel-Hamid et $a .^{16}$ observed that unilateral ligation of the internal iliac artery had a negative effect on haemodynamic parameters in impotent recipients compared to unilateral end-to-side anastomosis to the external

Table 2 Erectile performance before and 3 months after renal transplant $(\boldsymbol{N}=22)$

\begin{tabular}{|c|c|c|c|}
\hline & Baseline & 3 months after transplant & P value ${ }^{a}$ \\
\hline IIEF-EF (mean士s.d.) & $22.50 \pm 7.09$ & $18.09 \pm 6.33$ & 0.01 \\
\hline \multicolumn{4}{|l|}{ IIEF-EF ${ }^{18}, n / N(\%)$} \\
\hline Absent ED (EF score, 26-30) & $12 / 22(54.5)$ & $2 / 22(9.1)$ & 0.01 \\
\hline Mild ED (EF score, 17-25) & $5 / 22(22.7)$ & $13 / 22(59.1)$ & 0.03 \\
\hline Moderate ED (EF score, 11-16) & $3 / 22(13.6)$ & 4/22 (18.2) & 0.99 \\
\hline Severe ED (EF score, $\leqslant 10)$ & 2/22 (9.1) & 3/22 (13.6) & 0.99 \\
\hline Erectile grades $^{20}$ (mean \pm s.d.) & $3.11 \pm 0.91$ & $2.79 \pm 0.84$ & 0.06 \\
\hline Erectile grades $<3, n / N(\%)$ & $7 / 22(31.8)$ & $10 / 22(45.5)$ & 0.56 \\
\hline
\end{tabular}

Abbrevations: ED, erectile dysfunction; EF, erectile function; IIEF-EF, International Index of Erectile Function-Erectile Function.

${ }^{a}$ Two-tailed paired $t$-test, statistically significant values are reported in bold. 
Table 3 Comparison of CDUS haemodynamic parameters before and 3 months after renal transplant $(N=23)$

\begin{tabular}{|c|c|c|c|}
\hline & CDUS pre-transplant & CDUS post-transplant & P value \\
\hline $\mathrm{PSV}<30 \mathrm{~cm} \mathrm{~s}^{-1}, \mathrm{n} / \mathrm{N}(\%)$ & 6/23 (26) & 8/23 (35) & 0.73 \\
\hline PSV $<30 \mathrm{~cm} \mathrm{~s}^{-1}$, transplant side, $n / N(\%)$ & $4 / 23(17)$ & $6 / 23(26)$ & 0.70 \\
\hline PSV $($ mean \pm s.d. $)\left(\mathrm{cm} \mathrm{s}^{-1}\right)$ & $49.40 \pm 17.06$ & $44.03 \pm 13.48$ & 0.10 \\
\hline PSV right side (mean \pm s.d.) $\left(\mathrm{cm} \mathrm{s}^{-1}\right)$ & $50.72 \pm 16.45$ & $42.94 \pm 13.63$ & 0.04 \\
\hline PSV transplant side $\left(\right.$ mean \pm s.d.) $\left(\mathrm{cm} \mathrm{s}^{-1}\right)$ & $52.01 \pm 19.91$ & $42.60 \pm 18.77$ & 0.01 \\
\hline \multicolumn{4}{|l|}{$\begin{array}{l}\text { Postoperative EF domain score reduction }{ }^{\mathrm{b}} \text { : } \\
\quad \geqslant 4 \text { points }(N=12)\end{array}$} \\
\hline PSV transplant side (mean \pm s.d.) & $51.09 \pm 15.47$ & $41.97 \pm 19.06$ & 0.02 \\
\hline \multicolumn{4}{|l|}{$<4$ points $(N=10)$} \\
\hline PSV (mean \pm s.d.) & $51.39 \pm 21.72$ & $42.78 \pm 13.85$ & 0.14 \\
\hline PSV transplant side (mean \pm s.d.) & $55.33 \pm 24.68$ & $43.67 \pm 20.35$ & 0.09 \\
\hline $\mathrm{EDV}>5 \mathrm{~cm} \mathrm{~s}^{-1}, \mathrm{n} / \mathrm{N}(\%)$ & $12 / 23(52)$ & $11 / 23(48)$ & 0.98 \\
\hline EDV $($ mean \pm s.d. $)\left(\mathrm{cm} \mathrm{s}^{-1}\right)$ & $5.98 \pm 3.07$ & $5.62 \pm 3.17$ & 0.74 \\
\hline RI left side (mean) $\left(\mathrm{cm} \mathrm{s}^{-1}\right)$ & 0.88 & 0.88 & 0.76 \\
\hline RI right side (mean) $\left(\mathrm{cm} \mathrm{s}^{-1}\right)$ & 0.90 & 0.89 & 0.71 \\
\hline
\end{tabular}

Abbreviations: CDUS, colour Doppler ultrasound; EDV, end diastolic velocity; EF, Erectile Function; PSV, peak systolic velocity; RI, resistance index.

${ }^{a} t$-test, statistically significant values are reported in bold.

${ }^{\mathrm{b}}$ One patient failed to complete the 3-month postoperative IIEF-EF domain questionnaire.

iliac artery. As a result, end-to-side anastomosis of the graft to the external iliac artery was advocated in order to avoid the risk of ED in patients with compromised pelvic blood flow. ${ }^{14}$ In a prospective study conducted on 50 patients revascularized with end-to-end anastomosis to the right internal iliac artery, the average dynamic PSVs were found significantly reduced after $\mathrm{KT}$ when compared with preoperative values, but they were always above the $30 \mathrm{~cm} \mathrm{~s}^{-1}$ threshold that excludes cavernosal arterial insufficiency. ${ }^{14}$ These findings imply that haemodynamic changes occurring after unilateral exclusion of the internal iliac artery should exert negligible effects on erectile function in the presence of normal baseline PSVs, and they confirm earlier experimental data in canine models in which occlusion of one hypogastric artery did not produce significant changes to the cavernosal arterial supply. ${ }^{21}$ Similar conclusions were drawn in a recent Chinese study on internal iliac artery KT recipients. ${ }^{22}$ To the best of our knowledge, a comparative evaluation of haemodynamic parameters before and after KT onto the external iliac artery has not been carried out. The adverse effect on postoperative sexual function, even when the graft is anastomosed to the external iliac artery, ${ }^{12}$ supports the need to rule out significant CDUS changes at the cavernosal arteries and constituted our primary study end point. The overall mean PSV values for both cavernosal arteries did not significantly decrease after KT in our series $(P=0.1)$. When comparisons were made for the cavernosal artery corresponding to the side of the graft, the postoperative PSV was significantly reduced compared to the preoperative value $(P=0.01)$. A reduction of the same magnitude in the PSV before and after KT was recorded at the right cavernosal artery. This observation may be explained by the fact that the majority of KTs were right-sided. In only two out of 23 patients did PSV changes translate into clinically meaningful $\left(<30 \mathrm{~cm} \mathrm{~s}^{-1}\right)$ reductions of PSVs. Similar to the study of El-Bahnasawy et al., ${ }^{14}$ the average postoperative PSV values remained well above the normality threshold, even at the transplant site. This finding implies that while a haemodynamic effect exists, it is probably unlikely to account for the detrimental effect of
KT on sexual function. In view of the rather arbitrary definition of the normal cut-off values for PSV, ${ }^{23}$ the potential impact on erectile function exerted by reductions in the PSV within the normality threshold remains to be proved. Significant changes $(P=0.02)$ in PSVs before and after surgery were also observed in the subgroup of patients reporting a clinically meaningful $(>4$ points) reduction in the EF domain of the IIEF as compared to those patients who did not. Taken together, the findings on postoperative PSVs from our study and from the El-Bahnasawy's study ${ }^{14}$ do not seem to support the conclusion of Abdel Hamid et al. ${ }^{16}$ of a protective role of anastomosis of the graft to the external iliac artery on the blood supply to the penis. According to our results, end-to-side anastomosis to the external iliac artery cannot be viewed as providing a greater protective factor against ED than anastomosis carried out at the internal iliac artery. Interestingly, a recent prospective study that randomized $38 \mathrm{KT}$ recipients to undergo internal or external iliac artery anastomosis demonstrated no differences in postoperative erectile function, assessed by IIEF, between the two groups and concluded that both technical procedures are safe. ${ }^{24}$

Similar to the El-Bahnasawy's study, ${ }^{14}$ the EDV, a parameter suggestive of caverno-occlusive dysfunction when exceeding the $5 \mathrm{~cm} \mathrm{~s}^{-1}$ threshold, remained unaltered after KT. Abdel Hamid ${ }^{16}$ observed that impotent KT recipients had significantly higher EDVs than transplanted patients with normal sexual function and hypothesized a cyclosporine-based immunosuppressive regimen as one potential causative factor in view of its ability to promote fibrosis. ${ }^{25}$ In our study, a 2-week postoperative period was probably too short to detect haemodynamic changes that may have been promoted by immunosuppressive therapy. Notably, only one of our patients received a cyclosporine-based immunotherapeutic regimen. A negative effect of the immunosuppressive regimen on the 3-month IIEF cannot be excluded in our series.

With regard to hormonal changes, no statistical differences were found in the serum levels of testosterone or prolactin. In the literature 
there is no consensus on hormonal variations after renal transplantation. Akbari et al. ${ }^{26}$ found that hormonal levels in patients on haemodialysis improved after transplantation and returned to nearly normal, in contrast to the results of Shamsa et al., ${ }^{7}$ who described significant decreases in mean testosterone and prolactin levels after KT.

The limitations of the current study reside in the short follow-up. The 3-month interval was deliberatively chosen to avoid the potential interference of ED treatment in our IIEF evaluation at a later followup. Serial assessments with the IIEF, hormone profiles and CDUS parameters at later intervals could provide useful information about the pathogenesis of post-KT ED. A larger sample size may have allowed more meaningful results.

In conclusion, $\mathrm{KT}$ at the external iliac artery is associated with early worsening of erectile function. Testosterone and prolactin levels do not seem to account for these changes. Kidney grafts anastomosed at the external iliac artery cause significant reductions in penile arterial inflow, which remains, however, well within the normal range. PSV changes resemble those previously reported with grafts anastomosed at the internal iliac artery, suggesting that the type of artery chosen for KT plays little role in cavernosal artery haemodynamics. It remains to be proved whether haemodynamic changes of PSVs within the normality threshold may affect erectile function.

\section{AUTHOR CONTRIBUTIONS}

PG, BF and PS have made substantial contributions to conception and design of the study. ET, FF and EL contributed to acquisition of data. PG, MO and CF dealt with the analysis and interpretation of data. PG and MO have been involved in drafting the manuscript. PG, BF and AT have given final approval of the version to be published.

\section{COMPETING FINANCIAL INTERESTS}

The authors declare no competing financial interests.

\section{ACKNOWLEDGMENTS}

The authors are very grateful to all Medical and Nurse staff members of the division of Nephrology and Kidney Transplant of the Maggiore della Carità Hospital, Novara Italy.

1 NIH consensus conference. Impotence, NIH consensus development panel on impotence. JAMA 1993; 270: 83-90.

2 Navaneethan SD, Vecchio M, Johnson DW, Saglimbene V, Graziano G et al. Prevalence and correlates of self-reported sexual dysfunction in CKD: a meta-analysis of observational studies. Am J Kidney Dis 2010; 56: 670-85.
3 Rosas SE, Joffe M, Franklin E, Strom BL, Kotzker W et al. Prevalence and determinants of erectile dysfunction in hemodyalisis patients. Kidney Int 2001; 59 . 2259-66.

4 Kaufman JM, Hatzichristou DG, Mulhall JP, Fitch WP, Goldstein I. Impotence and chronic renal failure: a study of the hemodynamic pathophysiology. J Urol 1994; 151: 612-8.

5 Mehrsai A, Mousavi S, Nikoobakht M, Khanlarpoor T, Shekarpour L et al. Improvement of erectile dysfunction after kidney transplantation. J Urol 2006; 4: 240-4.

6 Tavallaii SA, Mirzamani M, Behzadi AH, Assari S, Vishteh HR et al. Sexual function: a comparison between male renal transplant recipients and hemodialysis patients. J Sex Med 2009; 6: 142-8.

7 Shamsa A, Motavalli SM, Aghdam B. Erectile function in end-stage renal disease before and after renal transplantation. Transplant Proc 2005; 37: 3087-9.

8 Nassir A. Sexual function in male patients undergoing treatment for renal failure: a prospective view. J Sex Med 2009; 6: 3407-14.

9 El-Bahnasawy MS, El-Assmy A, El-Sawy E, Ali-El Dein B, Shehab El-Dein AB et al. Critical evaluation of the factors influencing erectile function after renal transplantation. Int J Impot Res 2004; 16: 521-6.

10 Barroso LVS, Miranda EP, Cruz NI, Medeiros MAS, Araujo ACO et al. Analysis of sexual function in kidney transplanted men. Transplant Proc 2008; 40: 3489-91.

11 Malavaud B, Rostaing L, Rischmann P, Sarramon JP, Durand D. High prevalence of erectile dysfunction after renal transplantation. Transplantation 2000; 69: 2121-4.

12 Mirone V, Longo N, Fusco F, Verze $\mathrm{P}$, Creta $\mathrm{M}$ et al. Renal transplantation does not improve erectile function in hemodyalised patients. Eur Urol 2009; 56: 1047-54.

13 Espinoza R, Gracida C, Cancino J, Ibarra A. Prevalence of erectile dysfunction in kidney transplant recipients. Transplant Proc 2006; 38: 916-7.

14 El-Bahnasawy MS, El-Assmy A, Dawood A, Abobieh E, Ali-El Dein B et al. Effect of the use of internal iliac artery for renal transplantation of penile vascularity and erectile function: a prospective study. J Urol 2004; 172: 2335-9.

15 Hefty TB. Complications of renal transplantation: the practising urologist's role. AUA Update 1991; 10(lesson 8): 58-63.

16 Abdel-Hamid IA, Eraky I, Fouda MA, Mansour OE. Role of penile vascular insufficiency in erectile dysfunction in renal transplant recipients. Int J Impot Res 2002; 14: 32-7.

17 Nehra A, Kumar R, Ramakumar S, Myers RP, Blute ML et al. Pharmacoangiographic evidence of the presence and anatomical dominance of accessory pudendal artery(s). J Urol 2008; 179: 2317-20.

18 Cappelleri JC, Rosen RC, Smith MD, Mishra A, Osterloh IH. Diagnostic evaluation of the erectile function domain of the international index of erectile function. Urology 1999; 54: 346-51.

19 Gontero P, Fontana F, Bagnasacco A, Panella M, Kocjancic E et al. Is there an optimal time for intracavernous prostaglandin E1 rehabilitation following nonnerve sparing radical prostatectomy? Results from a hemodynamic prospective study. J Urol 2003; 169: 2166-9.

20 Virag R, Frydman D, Legman M, Virag H. Intracavernous injection of papaverine as a diagnostic and therapeutic method in erectile failure. Angiology 1984; 35: 79-87.

21 Aboseif SR, Breza J, Orvis BR, Lue TF, Tanagho EA. Erectile response to acute and chronic occlusion of the internal pudendal and penile arteries. J Urol 1989; 141: 398-402.

22 Ji ZG, Tian Y, Tang YW, Guo HB, Zhang L et al. Influence of the interruption of arteria iliaca interna distal end on penile vascularity and erectile function in male renal transplant recipients. Zhonghua Nan Ke Xue 2010; 16: 341-4.

23 Benson CB, Aruny JE, Vickers MA. Correlation of duplex sonography with arteriography in patients with erectile dysfunction. AJR Am J Roentgenol 1993; 160: 71-3.

24 Matheus WE, Reis LO, Ferreiura U, Mazzali M, Denardi F et al. Kidney transplant anastomosis: internal or external iliac artery? J Urol 2009; 6: 260-6

25 Shihab FS, Yi H, Bennett WM, Andoh TF. Effect of nitric oxide modulation on TGFbetal and matrix proteins in chronic cyclosporine nephrotoxicity. Kidney Int 2000; 58: 1174-85.

26 Akbari F, Alavi M, Esteghamati A, Mehrsai A, Djaladat $\mathrm{H}$ et al. Effect of renal transplantation on sperm quality and sex hormone levels. BJU Int 2003; 92: 281-3. 